\title{
Pflegewissenschaft und Praxis vernetzen
}

Prof. Dr. Stefanie Seeling ist Sprecherin der Sektion Onkologische Pflege der DGP. An der Hochschule Osnabrück hat sie eine Professur für Pflegewissenschaft im Bachelorstudiengang Pflege dual inne.

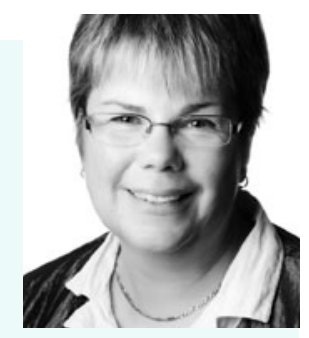

\section{HEILBERUFE: Frau Professor Seeling, welche Ziele verfolgt die Sektion Onkologische Pflege der DGP?}

Seeling: Die Sektion ist eine von zwölf Arbeitsgruppen der Deutschen Gesellschaft für Pflegewissenschaft, thematisch reiht sie sich in die Klinische Pflege ein. Die Mitglieder der Sektion forschen im Fachgebiet Onkologie. Ziel ist es, pflegewissenschaftliche Forschung und Praxis miteinander zu vernetzen, pflegewissenschaftlich gestützte Konzepte für Pflegende in der Onkologie zu generieren und Implementierungsmöglichkeiten aufzuzeigen. Ein weiteres Ziel ist es, Kooperationen zu Einrichtungen und Organisationen mit einem komplementären Ansatz aufzubauen und Synergien aus diesen spezifischen Angeboten für die onkologische Pflege zu nutzen.

Gibt es aktuell einen Forschungsschwerpunkt? Seeling: Gegenwärtig arbeiten wir an dem Thema Hautstörungen als Nebenwirkung von Target- und Chemotherapie. Hautstörungen sind in der Pflegepraxis weit verbreitet und nehmen offensichtlich zu. In der Pflege gibt es zwar ein großes Erfahrungswissen zum Umgang mit Hautstörungen, aber evidenzbasierte Pflegestandards fehlen. Dabei stellen Hautstörungen für Patienten eine elementare Einschränkung der Lebensqualität dar.

Aktuell laufen Literaturrecherchen, um den Stand der Forschung zu den verschiedenen Hautstörungen und deren Therapien abzubilden. Wir eruieren Informationsschriften zum Thema und stellen Kontakte her zu Personen, die ebenfalls diesen Forschungsschwerpunkt im nationalen und internationalen Kontext haben.

In der Pflegepraxis erarbeiten Mitglieder der Sektion eine Fotodokumentation der wahrgenommen Phänomene. Weiter ist geplant, eine Befragung zur Thematik in Kliniken in Norddeutschland durchzuführen. Dafür entwickeln wir gerade einen Fragebogen.Zudemmöchtenwireinpflegewissenschaftliches, evidenzbasiertes Versorgungskonzept entwickeln, in dem auch die Ergänzung der onkologischer Fachkrankenpflege durch komplementäre Pflegemaßnahmen bei Hautstörungen vorgesehen ist.

Eine Präsentation der Ergebnisse ist für 2014/2015 geplant.
Sollten onkologische Fachkrankenpfleger akademisch ausgebildet sein?

Seeling: Die Sektion vertritt die Position, dass die onkologische Pflege zukünftig eine weitere Spezialisierung erfahren soll und im Sinn eines klinischen Masters Kompetenzen durch ein Studium erworben werden müssten. Die Zunahme von hochkomplexen Versorgungssituationen bei onkologischen Patienten zieht diesen Bedarf nach sich.

Die Diskussion über Delegation und Substitution ärztlicher Aufgaben ist auch in der Onkologie sehr gegenwärtig. In der Praxis wird vieles von dem, was da diskutiert wird, schon praktiziert. Für akademisch spezialisierte Pflegende könnten diese Tätigkeiten konkret definiert werden und in ihren Verantwortungsbereich übergehen. So kann das, was bisher meist in einem stillschweigenden Abkommen läuft, transparent und kompetenzbasiert organisiert werden. Damit wäre auch die Voraussetzung geschaffen, pflegewissenschaftliche Forschungsergebnisse in der Praxis umzusetzen. Langfristig sollten diese Überlegungen bei der Konzeption von Masterstudiengängen berücksichtigt werden und dann eine Ergänzung der bewährten Fachweiterbildung in der onkologischen Pflege sein.

\section{Das Interview führte Heike Ottow}

\section{Sektion Onkologische Pflege}

Die Sektion Onkologische Pflege wurde 2005 als eine Sektion der DG-Pflegewissenschaft gegründet. Mitglieder sind Pflegepraktiker, die in der onkologischen Pflege tätig sind, aber auch Pflegewissenschaftler. Alle haben einen Arbeits-, Forschungs- oder Promotionsschwerpunkt in der onkologischen oder palliativen Pflege. Die Prozesse in der Sektion laufen arbeitsteilig. Die Mitglieder treffen sich viermal im Jahr. Interessierte Kollegen sind herzlich willkommen.

www.dg-pflegewissenschaft.de 